\title{
La violence dans les collèges au XVIIIe siècle
}

Violence in 18th century schools

Ausschreitungen in höheren Schulen im 18. Jahrhundert

La violencia en los colegios en el siglo XVIII

\section{Philippe Marchand}

\section{(2) OpenEdition}

\section{Journals}

Édition électronique

URL : https://journals.openedition.org/histoire-education/546

DOI : 10.4000/histoire-education.546

ISSN : 2102-5452

Éditeur

ENS Éditions

Édition imprimée

Date de publication : 1 avril 2008

Pagination : 67-82

ISBN : 978-2-7342-1117-4

ISSN : 0221-6280

Référence électronique

Philippe Marchand, «La violence dans les collèges au XVIIle siècle», Histoire de l'éducation [En ligne],

118 | 2008, mis en ligne le 01 janvier 2013, consulté le 20 mai 2021. URL : http://

journals.openedition.org/histoire-education/546; DOI : https://doi.org/10.4000/histoire-education.546

(c) Tous droits réservés 


\title{
La violence dans les collèges au XVIII ${ }^{\mathrm{e}}$ siècle $^{1}$
}

\author{
Philippe MARCHAND
}

Depuis les travaux pionniers du père François de Dainville ${ }^{2}$ et de Philippe Ariès $^{3}$, l'historiographie des collèges d'Ancien Régime s'est considérablement enrichie $^{4}$. Si les approches se sont multipliées, il n'en subsiste pas moins quelques zones d'ombre, et en particulier tout ce qui touche à la violence, violence écolière et violence de leurs maîtres, à l'intérieur des collèges. De 1976 à 2006, la Bibliographie de l'histoire de l'éducation française publiée par la revue Histoire de l'éducation ${ }^{5}$ ne mentionne qu'une référence consacrée à des manifestations de violence dans l'enceinte d'un collège ${ }^{6}$. Il faut dire que les traces laissées par de

1 Cet article est une version revue d'une communication publiée dans le Bulletin de la Commission historique du Nord, XLVI, 1991-1992, p. 23-40.

2 François de Dainville, L'Éducation des Jésuites XVIe-XVIII siècles, Paris, Les Éditions de Minuit, 1978 (réédition par Marie-Madeleine Compère des articles de François de Dainville consacrés à l'histoire de l'éducation).

3 Philippe Ariès, L'Enfant et la vie familiale sous l'Ancien Régime, Paris, Plon, 1960. Dans son étude de l'évolution de l'enfance, l'auteur fait une large place à l'histoire de la scolarisation.

4 Parmi les nombreux ouvrages publiés au cours des trente dernières années, on peut citer, dans l'ordre chronologique : Jean de Viguerie, L'Institution des enfants. L'éducation en France $16^{e}$ - $18^{e}$ siècles, Paris, Calmann-Lévy, 1976 ; François Lebrun, Marc Venard, Jean Quéniart, De Gutenberg aux Lumières (1480-1789), t. 2 de l'Histoire générale de l'enseignement et de l'éducation en France, Paris, Nouvelle Librairie de France, 1981 ; Marie-Madeleine Compère, Dominique Julia, Les Collèges français $16^{e}-18^{e}$ siècles. Répertoire 1 - France du Midi ; Répertoire 2 - France du Nord et de l'Ouest ; Répertoire 3 - Paris, Paris, INRP/CNRS, 1984, 1988, 2002 ; Marie-Madeleine Compère, Du collège au lycée (1500-1850), Paris, Gallimard/Julliard, 1985 (coll. Archives).

5 Cette bibliographie fait désormais l'objet d'une publication électronique, à l'adresse www.inrp.fr/she/bhef/

6 Jean Reyrat, "Un jeune Confolentais expulsé du collège des Jésuites de Limoges en 1653 ", Les Amis du Vieux Confolens, 65, 1998, p. 13-14. L'auteur publie le jugement rendu à l'encontre de plusieurs écoliers de rhétorique coupables d'avoir perturbé violemment les classes à deux reprises. 
telles manifestations sont rares. Les récits autobiographiques en recèlent parfois. Mais la mémoire sélective de leurs auteurs en idéalise et en transfigure quelquefois le souvenir. Dans leur description des situations conflictuelles opposant les élèves entre eux, ou les élèves aux maîtres, les anciens collégiens se donnent toujours le beau rôle. Faut-il rappeler Marmontel entraînant les rhétoriciens du collège de Mauriac dans une révolte victorieuse contre le préfet des études ${ }^{7}$ ? Ou la rébellion de Chateaubriand contre un régent du collège de Dol, à qui il lance un Macte animo, generose puer! (Courage, généreux enfant !) qui lui permet d'obtenir un pardon magnanime ${ }^{8}$ ? Sur la violence entre les élèves, les récits autobiographiques sont étrangement silencieux. Restent les archives, dont il faut d'emblée souligner les limites. En effet, les situations où se manifestent la turbulence, voire la violence écolière, d'une part, l'emportement et la rudesse des maîtres, de l'autre, sont soigneusement occultées par les responsables des collèges, comme le montrent les deux exemples suivants ${ }^{9}$. Le 8 janvier 1778, un "tumulte " éclate au collège de Lille, " parce qu'il n'y avait point eu congé ce jour là ". Les élèves refusent de rentrer en classe. La " révolte " semble n’avoir duré que quelques heures. On n'en sait guère plus, car le bureau d'administration ne juge pas utile de publier les rapports du principal et du sous-principal dans le registre des délibérations. Autre exemple : en 1779, un régent du même établissement, porté sur la boisson, se voit reprocher sa brutalité envers les élèves. En dépit de nombreux rappels à l'ordre, son comportement ne s'améliore guère et son renvoi est prononcé sans autre précision que celle de la peine.

Pour que soit levé le secret des comportements et des pratiques quand se déchaînent à l'intérieur des collèges la violence des élèves et celle de leurs maîtres, il faut que les incidents dégénèrent au point de provoquer l'intervention de la justice ${ }^{10}$. Tel est le cas dans deux affaires qui se sont déroulées, la première au collège du Quesnoy en $1757^{11}$, la seconde au collège de Bavay en $1758^{12}$. Le collège du Quesnoy est un collège municipal ouvert en 1676. Dans les années 1750-1760,

7 Jean-François Marmontel, Mémoires d'un père pour servir à l'instruction de ses enfants, Paris, Costes et Compagnie, 1819.

8 François-René de Chateaubriand, Mémoires d'Outre-tombe, Paris, Gallimard, 1951 (Bibliothèque de la Pléiade), t. 1, p. 58-60.

9 Philippe Marchand, Recherches sur l'histoire de l'enseignement au XVIII' siècle. Le collège de Lille, $1^{\text {er }}$ auril 1765- 10 nivôse an $V$ (30 décembre 1796), Thèse de $3^{\mathrm{e}}$ cycle, 1971, Archives départementales (désormais AD) du Nord, 3 J 321, 1 et 2, p. 384 et 684.

10 C'est le cas au collège de Limoges, où une plainte est déposée par le père procureur des jésuites.

11 AD Nord, C 7121.

12 AD Nord, C 7122. 
il offre le cours complet des études de la sixième à la rhétorique ; trois régents se partagent les classes. L'établissement possède un pensionnat dont l'essor est certain au milieu du siècle. Les élèves sont au nombre de 150 en 1676 ; on en compte 300 à la fin du siècle ${ }^{13}$. Le collège de Bavay, ouvert en 1633, est tenu par les pères de l'Oratoire. Au milieu du XVIII siècle, les élèves peuvent, comme au Quesnoy, y suivre le cours complet des études. Le personnel comprend un supérieur, un professeur de rhétorique, quatre régents de la seconde à la sixième, et deux préfets chargés de la surveillance des études et du pensionnat. Fort prospère, celui-ci fournit une bonne partie des effectifs, évalués à 400 élèves en $1789^{14}$.

\section{I - Les faits}

La première affaire est une rixe tragique opposant deux élèves du collège du Quesnoy dans les premiers jours de février 1757. Deux lettres, l'une de Delegove, subdélégué de l'intendant du Hainaut au Quesnoy, l'autre de Blair de Boisemont, intendant du Hainaut, permettent d'en retracer le déroulement ${ }^{15}$. Les pensionnaires sont rassemblés dans le chauffoir pour le déjeuner. Paul Pasqual, élève de rhétorique âgé de dix-sept ans, jette une pomme cuite qui vient s'écraser contre la buse du poële. Des morceaux rebondissent et atteignent le chapeau de Florent Maximilien de Lattre, élève de sixième, âgé de treize ans ${ }^{16}$. Celui-ci interpelle d'une façon désobligeante - une insulte sans doute ? - Paul Pasqual, qui riposte en le souffletant. Florent de Lattre le saisit alors par le cou et le frappe d'un coup de couteau qui lui coupe le sifflet. Les deux lettres ne disent rien de la nature et de l'origine du couteau utilisé par le jeune meurtrier. Une remarque du subdélégué de l'intendant du Hainaut laisse à penser qu'il ne s'agit pas de la pièce du service de table que tout pensionnaire doit apporter dans ses bagages, mais plutôt d'un

13 Philippe Marchand, "Le Quesnoy Collège d'humanités " dans Marie-Madeleine Compère, Dominique Julia, Les collèges français $16^{e}-18^{e}$ siècles Répertoire 2-France du Nord et de l'Ouest, op. cit., p. 424-426.

14 Ibid, p. 88-91.

15 AD Nord C 7121 : lettre de Delegove, subdélégué au Quesnoy, à Blair de Boisemont, intendant du Hainaut, 5 février 1757 ; minute de la lettre de Blair de Boisemont, intendant du Hainaut, au comte de Saint-Florentin, secrétaire d'État, 12 mai 1757. Blair de Boisemont a été intendant du Hainaut de 1754 à 1764 .

16 Né le 23 juin 1743 de Roger Florent de Lattre, seigneur de Feignies, et d'Alexandrine Levalliant, Florent Maximilien a deux frères aînés issus d'un premier mariage paternel, dont l'un sert en Espagne, l'autre dans les armées de l'impératrice Marie-Thérèse. La fortune personnelle du jeune meurtrier est fort médiocre. Comme le veut la coutume du Hainaut, la terre de Feignies, dont jouit son père, revient aux deux fils issus du premier mariage. Il ne lui reste " presque d'autres ressources que le partage qui lui reviendra dans le peu de bien que sa mère a porté en mariage ". Florent de Lattre est noble. En revanche, Paul Pasqual, orphelin, est roturier. Son père était greffier de la maîtrise des eaux et forêts du Quesnoy. 
coutelas introduit dans le collège par de Lattre en violation du règlement. Le meurtrier s'enfuit immédiatement du collège. Les correspondances échangées entre le subdélégué et l'intendant, et entre l'intendant et le comte de Saint-Florentin, secrétaire d'État chargé du suivi de l'affaire, montrent qu'une information judiciaire est ouverte, dans laquelle la mère de Paul Pasqual se porte partie civile. En mai 1757, le père de Florent de Lattre sollicite des lettres de rémission pour son fils ${ }^{17}$. Le sort de cette demande, ainsi que son dénouement judiciaire, restent inconnus.

La seconde affaire est une agression d'un élève du collège de Bavay, Gilles Levent, âgé de dix-sept ans, contre le père de Glines, supérieur de l'établissement Les faits nous sont connus par la correspondance échangée entre l'intendant Blair de Boisemont, son subdélégué à Bavay Hennet et le comte de Saint-Florentin, et par les Lettres d'ester à droit de Gilles Levent ${ }^{18}$. Leur date exacte reste inconnue, mais on peut les situer pendant le mois d'octobre 1758, au lendemain, donc, de la rentrée des classes. Les élèves de la classe de poésie (seconde) sont réunis dans une salle du collège appelée Museum. En l'absence de leur régent - on en ignore le motif -, ils sont placés sous la surveillance d'un des leurs " faisant fonction de censeur ". L'écolier investi de cette fonction dénonce un dénommé Pierre Prévot au préfet et au sous-préfet du collège. Motif invoqué par le " censeur" : la " pétulance " de son condisciple. Le père Cordier, sous-préfet du collège, décide, pour le punir, de le faire fouetter.

À l'annonce " de ce châtiment humiliant auquel ne sont point assujettis les élèves de seconde pour une faute aussi légère ", les collégiens protestent " contre ce renversement des usages et des privilèges qu'avaient les élèves de cette classe de se regarder comme exempts de cette punition ". Gilles Levent, âgé de dix-sept ans, se fait le porte-parole de ses condisciples pour faire valoir leurs droits. Débordé, le père Cordier en appelle au père de Glines et accuse Gilles Levent d'être le meneur

$17 \mathrm{AD}$ Nord C 7121 : demande d'avis sur la suite à donner à la demande de lettres de rémission par le père de Florent de Lattre, 24 avril 1757. Les lettres de rémission sont accordées par le roi pour les crimes paraissant excusables attendu les circonstances, comme les homicides involontaires ou la nécessité d'une légitime défense.

18 AD Nord C 7122 : minute de la lettre de Blair de Boisemont, intendant du Hainaut, au comte de SaintFlorentin, secrétaire d'État, 27 janvier 1759 ; lettre de Hennet, subdélégué à Bavay, au comte de SaintFlorentin, secrétaire d'État, 18 janvier 1759 ; lettre du comte de Saint-Florentin, secrétaire d'État, à Blair de Boisemont, intendant du Hainaut, Versailles, 9 février 1759. Les Lettres d'ester à droit pour Gilles Levent sont publiées en annexe à cet article. Les lettres d'ester à droit permettent de poursuivre une action en justice. 
du tumulte ${ }^{19}$. Alertés par le bruit, les autres régents du collège accourent et tentent de maîtriser le collégien, qui s'est réfugié sur une table. Le père de Glines parvient à s'en saisir par la jambe, tente de le faire tomber, le frappe durement contre la table et le blesse en plusieurs endroits. Dans la bousculade, Gilles Levent, "qui avait inutilement demandé à sortir du collège ainsi qu'une grande partie des écoliers ", frappe le père de Glines d'un coup de couteau à l'épaule droite, parvient à sortir de la classe, escalade les murs du collège et se cache dans une maison voisine, où ses blessures sont soignées. Puis il se réfugie dans l'auberge que ses parents tiennent à Bavay. Le père de Glines n'est que légèrement blessé et peut continuer " ses fonctions ordinaires ". Cependant, décidé à faire un exemple, il porte l'affaire devant la justice. Le père du collégien, Pierre Levent, sollicite du roi le pardon du coupable. Mais, inquiet de la tournure prise par les événements, il envoie son fils chercher refuge dans le Hainaut autrichien voisin. La suite de l'affaire allait lui donner raison. En effet, en février 1759, les commissaires royaux chargés de l'examen de la requête de Pierre Levent la rejettent et décident de laisser la justice faire son œuvre. Le 3 mars 1759, le Parlement de Flandre condamne par contumace Gilles Levent à la peine des galères à perpétuité. Il obtient le pardon royal en 1770 et peut rentrer à Bavay. Il est alors âgé de vingt-neuf ans.

Ces deux affaires, différentes dans leur nature, possèdent cependant un certain nombre de points communs : elles présentent des situations d'affrontement et de violence, entre élèves et entre maîtres et élèves ; elles mettent en cause le personnel de direction et les régents des deux collèges ; elles provoquent l'intervention de la justice et de l'administration. Seuls, les documents produits par l'administration sont parvenus jusqu'à nous. Il serait intéressant de les compléter et de les croiser avec les pièces produites lors des procédures judiciaires : dépositions des protagonistes, des témoins. Les recherches menées pour les retrouver sont restées infructueuses.

19 Né le 21 octobre 1741, Gilles Levent est l'ainé de neuf enfants. Son père, désigné comme censier du comte de Louvignies dans les actes paroissiaux, est venu s'installer à Bavay pour y tenir une auberge. Le père Joseph Cordier, originaire de Lens dans le Hainaut autrichien, est entré dans l'Oratoire en 1750 et exerce la fonction de sous-préfet du collège de Bavay depuis janvier 1752. Le père de Glines, originaire de Mons (dans le Hainaut autrichien), est arrivé plus tard au collège. Son nom n'apparaît pas sur l'état du personnel dressé en 1757. 


\section{II - Des élèves livrés à eux-mêmes}

De nombreux travaux ont développé l'idée selon laquelle les régents et maîtres de quartier ou préfets des études exerçaient une surveillance continue sur les collégiens, et en particulier sur les pensionnaires ${ }^{20}$. Les textes normatifs et les prospectus des pensionnats ne cessent de signaler que les élèves ne sont jamais livrés à eux-mêmes ${ }^{21}$. Or, que montrent les affaires de Lattre et Levent ? Au collège de Bavay, les collégiens sont dans leur classe, placés sous la surveillance de l'un d'entre eux faisant fonction de censeur. Son autorité est sanctionnée par le droit qui lui est conféré de dénoncer les "pétulants " aux régents et/ou aux surveillants. Comme le signale Philippe Ariès, cette pratique est ancienne ${ }^{22}$. Elle se perpétue dans les collèges du nord de la France jusqu'à la fin de l'Ancien Régime. Le Règlement de discipline élaboré en 1768 par le Parlement de Flandre pour les collèges de son ressort prévoit, dans son article 18, la possibilité " de préposer un censeur qui tiendra note des pétulants pendant les heures d'étude ${ }^{23}$. Le Règlement établi en 1788 au collège de Bergues pour définir la fonction du préfet des études l'autorise à nommer " des censeurs sur le parc et dans les classes pour lui faire un rapport fidèle des contraventions qui s'y commettraient " ${ }^{24}$.

En revanche, au collège du Quesnoy, les pensionnaires sont seuls dans le chauffoir, livrés à eux-mêmes, pour y prendre leur déjeuner. Aucune mention d'une quelconque surveillance dans les rapports de l'intendant et de son subdélégué. On peut s'interroger sur cette situation. Correspond-elle à une volonté de responsabiliser les élèves en instaurant une auto-discipline ? Ne convient-il pas plutôt d'invoquer des problèmes d'ordre matériel, et en particulier la nécessité

20 Voir notamment : Philippe Ariès, L'Enfant et la vie familiale..., op. cit., p. 281-285 : "Cette surveillance constante devint aux XVI ${ }^{\mathrm{e}}$ et XVII ${ }^{\mathrm{e}}$ siècles un des principes essentiels de l'éducation scolaire " ; Georges Snyders, La Pédagogie en France aux XVII e et XVIII ${ }^{e}$ siècles, Paris, Presses universitaires de France, 1965 , p. 38-41 : "Toujours une présence. - Effectivement, l'élève n'est jamais seul, jamais il n'est laissé à luimême " ; J. de Viguerie, L'Institution des enfants..., op. cit., p. 236-237 : "Les jésuites et leurs émules ont mis au point un système de surveillance dont la perfection n'a jamais été égalée ".

$21 C f$., par exemple, AD Nord, D 548/10, Règlement pour le principal et les régents du collège de Dunkerque, 29 octobre 1764, art. 33 : "Le Principal aura soin que les régents conduisent les pensionnaires à l'heure précise aux exercices où la cloche les appelle, que pendant les études et récréations il y ait toujours un régent pour présider dans les chambres, et une personne de confiance pour y veiller dans les cours, dortoirs et lieux communs où lesdits pensionnaires pourraient se trouver".

Philippe Ariès, L'Enfant et la vie familiale..., op. cit., p. 281-285.

AD Nord, C 18797 : Règlement de discipline pour les collèges du ressort du Parlement de Flandres formé en l'exécution des lettres patentes de 1766 et 1767 portant confirmation des dits collèges, 1768 .

24 Charles de Croocq, "Histoire du collège de Bergues Saint-Winoc (1600-1923) ", Annales du Comité flamand de France, XXXIII, 1923, p. 416-418. 
d'assurer le fonctionnement du pensionnat avec un personnel limité en nombre ? Cette seconde hypothèse semble être la bonne quand on sait les difficultés financières dans lesquelles se débattent les collèges de Flandre et du Hainaut pendant le XVIII ${ }^{e}$ siècle. Le collège du Quesnoy connaît une réelle situation de sous-encadrement. Un principal et trois régents s'occupent d'un établissement offrant le cours complet des études de la sixième à la rhétorique et comprenant un pensionnat. L'essor du collège ne fait pas de doute au moment de l'affaire de Lattre. Les devis des travaux qu'on y fait pour le pensionnat en témoignent. En 1742, le pensionnat comprend douze chambres. Quatre ans plus tard, l'afflux de pensionnaires est tel que la ville décide de construire un bâtiment neuf offrant dix chambres supplémentaires, un chauffoir et une salle de classe supplémentaire. Le coût total de l'opération s'élève à 6300 livres. Les caisses de la ville sont vides, rendant impossible la rémunération de surveillants.

On notera également la facilité avec laquelle les deux collégiens parviennent à s'enfuir du collège.

\section{III - L'usage des verges}

L'affaire Gilles Levent montre que les régents et les surveillants ont toujours recours, dans la décennie 1750-1760, aux châtiments corporels pour punir ce qu'ils considèrent comme un manquement à la discipline. L'attitude des Oratoriens du collège de Bavay à l'égard de ces châtiments est cependant ambiguë. En les réservant aux élèves des classes de grammaire (de la sixième à la troisième) et en y renonçant pour les élèves des classes d'humanités (de la seconde à la rhétorique), ils semblent admettre leur inadaptation, au moins pour les élèves les plus âgés. Mais ce que les élèves considèrent comme une "règle " et un " privilège " n'est, aux yeux des enseignants, qu'un " usage " pouvant être rapporté selon les circonstances.

On lit la même ambiguïté dans le discours des autorités. Elles soulignent le " caractère humiliant " des verges appliquées " à un jeune écolier [...] se voyant devenir homme et la disproportion entre la légèreté de la faute et la gravité du châtiment ". Mais elles ne vont pas jusqu'à condamner formellement l'usage des verges.

Ces incertitudes perdurent jusqu'à la fin de l'Ancien Régime. Des voix s'élèvent dans le corps enseignant pour condamner le recours aux châtiments corporels et suggérer des peines de remplacement : pensum, privation de sortie... Hostile aux " coups " qui " abrutissent " les élèves, Gabriel de Garsignies, écolâtre du collège 
de Saint-Pierre de Lille, propose une échelle de sanctions allant des remontrances aux arrêts :

Il serait à désirer qu'on puisse conduire les jeunes gens par des principes de raison, d'honnêteté et de religion. Point de paroles dures, point de coups, on les abrutit : s'il faut les punir, que ce soit par la privation des plaisirs attachés à leur âge et par un certain air de froideur qui les déconcerte. Il serait mieux de les prendre par les sentiments. S'ils ne sont pas sensibles aux remontrances, qu'ils soient privés de récréations : que la plus forte punition soit d'être mis aux arrêts dans la chambre pendant une journée ${ }^{25}$.

Les parents aussi font entendre leur voix, en s'inquiétant de la rudesse de la discipline au collège de Lille. Ils vont jusqu'à menacer de retirer leurs enfants du pensionnat s'il n'y est pas mis bon ordre ${ }^{26}$.

Mais il y a la réalité quotidienne, c'est-à-dire des régents et des surveillants en butte à la turbulence écolière. Dans bien des situations, comme celle qu'affronte le père Cordier, la correction publique leur apparaît comme l'ultime recours susceptible d'imposer une autorité contestée. Les textes normatifs se montrent d'ailleurs bien équivoques sur la question des châtiments corporels. Que disent les règlements approuvés par l'assemblée de l'Oratoire de France en 1634, et devenus obligatoires pour tous les collèges oratoriens?

Le préfet et les régents s'abstiendront de blesser les écoliers par des paroles injurieuses : que jamais ils ne les frappent du pied, de la main, avec des livres, qu'ils les punissent des châtiments autorisés.

Que promet le novice avant d'être reçu définitivement dans l'Oratoire ?

Je me comporterai dans la correction comme un père et j'aimerai mieux être repris de Dieu d'une trop grande bonté que de trop de sévérité. Je ferai mon possible pour avoir leur confiance plutôt par amour et par douceur que par rigueur et plutôt par artifice que par justice. Et pour cela, je leur témoignerai un grand amour même lorsqu'il faudra les châtier, leur faisant connaître l'aversion que j'ai d'en venir là parce que je les aime tendrement. ${ }^{27}$

25 AD Nord, 16 G 520 : Gabriel de Garsignies, Le seul désir de faire refleurir le collège de Saint-Pierre, 1778.

26 Philippe Marchand, "Les parents et l'éducation dans les collèges de la France du Nord : des parents écrivent ", Livre et Lumières dans les Pays-Bas français de la Contre-Réforme à la Révolution, Colloque de Valenciennes, 1985, Cercle archéologique et historique de Valenciennes, t. X, vol. 8, p. 39-40. Un père écrit au principal : " J'aime mes enfants, vous devez le savoir, je veux leur donner de l'éducation, mais je ne veux pas qu'ils soient battus : on peut trouver tant d'autres façons de punir sans en venir aux coups, on me les dégoûterait et je n'en ferais plus rien. J'ose espérer... que vous voudrez donner des ordres en conséquence ".

27 Paul Lallemand, Histoire de l'éducation dans l'ancien Oratoire de France, Paris, E. Thorin, 1889, p. 226 et p. 356. 
Les règlements en vigueur dans les collèges séculiers sont tout aussi flous. On y parle de " corrections sages et modérées ", de " châtiments proportionnés aux fautes ", de la " prudence " à observer quand il faut punir. Il est conseillé aux régents de se concerter " lorsqu'il s'agira de corriger un étudiant pour de grandes fautes ". Et surtout, on leur recommande de toujours se couvrir de l'autorité du principal et de faire administrer les verges par le correcteur du collège ${ }^{28}$. Ce recours au correcteur, mis à l'honneur par les jésuites, à qui il était interdit d'exécuter tout châtiment corporel de leurs propres mains, ne semble pas en usage au collège de Bavay. Il était pourtant fréquent dans les collèges de Flandre et du Hainaut. Au collège du Cateau, les collégiens versent, le jour de la rentrée, douze patars pour rémunérer le correcteur, qui fait aussi office de balayeur ${ }^{29}$. Les administrateurs du collège de Cambrai, informés par les régents de l'impossibilité " de contenir les écoliers dans l'ordre prescrit pour leur meilleure éducation et obéissance sans qu'il y ait un cuistre pour les intimider et pour les punir ", leur donnent satisfaction. Le principal recrute un homme de peine chargé d'administrer les verges et de garder la porte d'entrée du collège ${ }^{30}$. Le chapitre de la collégiale Saint-Pierre de Lille alloue quarante livres " à un homme pour corriger les enfants du collège " ${ }^{31}$. Un régent du collège de Saint-Omer, Philippe Le Roux, décrit le cérémonial qui entoure la correction et doit impressionner les élèves :

En récréation, deux élèves s'étaient battus, je les priai de se rendre en la grande salle. Vous savez que c'est celle où se font les exécutions de la haute et basse justice. J'étais accompagné du sous-principal, des professeurs et d'un bon nombre de maîtres de quartier. Un oiseau de mauvais augure, le correcteur, décoré de toutes les marques de sa dignité, nous suivait d'assez près. Cet appareil insolite frappa tous les élèves ${ }^{32}$.

28 BM Saint-Omer, ms 1411, Règlement de discipline pour le collège français de Saint-Omer, 24 décembre 1768 ; Archives municipales de Bergues, CJ n 197, Règlement général pour le collège de Bergues SaintWinoc, 19 décembre 1766 ; Archives de l'État, Tournai, n 71, Règlement général pour la discipline des classes du collège de Lille, octobre 1780 ; id., Précis ou idée générale des devoirs et fonctions des maîtres de quartier du collège de Lille.

29 Charles Croix, Histoire du collège du Cateau-Cambrésis depuis son origine jusqu'en 1791, Paris, H. Champion, 1926, p. 63.

30 Durieux, Le Collège de Cambrai (1270-1882) d'après des documents inédits, Cambrai, 1882, p. 99.

31 E. Hautcoeur, Histoire de l'église et collégiale et du chapitre de Saint-Pierre de Lille, Paris, 1896-1899, t. 3, p. 283.

$32 C f$. Georges Delamotte et J. Loisel, Les Origines du lycée de Saint-Omer. Histoire de l'ancien collège 1565-1845, Calais, 1910, p. 237-240. Philippe Le Roux est l'auteur de Lettres sur la manière de former le caractère des jeunes gens, 1780 . 


\section{IV - Les enseignants condamnés par les autorités}

Les autorités administratives qui se saisissent des dossiers de Lattre et Levent sont unanimes dans leur condamnation de la conduite des enseignants. Le principal du collège du Quesnoy se voit d'abord reprocher d'avoir laissé les pensionnaires en possession de couteaux, ce qui est contraire au règlement. Le subdélégué de l'intendant du Hainaut souligne :

Le principal du collège a grand tort de souffrir que des enfants aient des couteaux puisque de tous temps ils ont été défendus aux étudiants.

Un arrêt du Parlement de Flandre interdit en effet aux collégiens d'être munis d'un couteau et punit " des galères celui qui en frappe quelqu'un ". Le Règlement d'étude et de discipline pour les collèges du ressort du parlement de Flandre de 1768 rappelle, dans son article 38, que " les principal, sous-principal et régents veilleront à ce que les écoliers ne se servent de ciseaux, couteaux, canifs ou autres instruments dangereux ". Le Règlement pour le collège de Dunkerque signale que les pensionnaires doivent déposer " leurs épées chez le portier sans pouvoir les conserver dans leurs chambres ". Mais au cours de l'instruction, l'accusation de négligence portée contre le principal du collège du Quesnoy n'est pas retenue. Ce qui lui est reproché, c'est d'avoir laissé les pensionnaires sans surveillance, ce qui est également contraire aux règlements. L'intendant écrit :

Je penserai donc que cet accident [...] ne peut être considéré que comme un de ces malheurs qui arrivent assez fréquemment lorsqu'on livre des jeunes gens à euxmêmes et qu'il n'y a personne d'autorité pour leur en imposer.

Dans l'affaire Levent, l'intendant du Hainaut n'est pas moins sévère avec l'ensemble du personnel du collège, dont il souligne l'incapacité à gérer une situation conflictuelle :

Le préfet, le sous-préfet aussi bien que le père de Glines ont beaucoup à se reprocher la conduite imprudente qu'ils ont tenue, $1^{\circ}$ ) en s'obstinant à vouloir faire punir par des fautes une faute légère en elle-même et d'avoir occasionné l'émeute qui s'est levée parmi les écoliers de Poésie ; $2^{\circ}$ ) le Supérieur de son côté pouvait facilement apaiser un tumulte en faisant grâce au nommé Prévot. De cette manière, il aurait rétabli la paix dans la chambre du poële sans donner atteinte à l'autorité.

Ce développement raturé dans la minute du rapport de l'intendant figure dans la version définitive adressée au secrétaire d'État, Saint-Florentin, puis remise aux commissaires chargés de l'examen de l'affaire. Saint-Florentin reprend l'argument 
de "l'imprudence qu'il a pu y avoir de la part des maîtres ". En décidant d'appliquer un châtiment manifestement disproportionné à une "faute aussi légère ", en refusant de faire preuve de mansuétude, ce qui en dernier ressort aurait peut-être permis de ramener le calme, les pères de Glines et Cordier ont manqué de clairvoyance et sont à l'origine du tumulte. Toutes ces remarques figurent à nouveau dans les Lettres d'ester à droit de Gilles Levent. L'aveuglement et la violence du supérieur et du sous-préfet du collège de Bavay sont ainsi officiellement reconnus comme la cause du geste de Gilles Levent.

Tout en accablant les enseignants, l'intendant du Hainaut et son subdélégué tentent de comprendre, voire de justifier le comportement des deux accusés. Pour Blair de Boisemont, la réaction du jeune de Lattre trouve son origine dans son statut social :

Il est assez naturel de penser qu'un jeune homme de famille noble, et d'ailleurs né vif et bouillant, au passé par-dessus toute considération, se soit cru tout permis pour se venger d'un affront qu'il a pu regarder, dans les préjugés où est élevée la jeune noblesse, comme une espèce de déshonneur venant de la part de quelqu'un d'une famille bourgeoise.

Guant à Gilles Levent, l'" effervescence " de l'âge justifie sa conduite aux yeux de l'intendant du Hainaut, dont le subdélégué développe, dans son exposé de l'affaire, une argumentation d'ordre psychologique fondée sur la problématique du passage de l'enfance à l'âge adulte :

C'est un jeune écolier âgé de seize ans, peu instruit des ordonnances et de leur sévérité, qui, se voyant devenir un homme et honteux de souffrir la verge, emploie dans un premier moment ce qui se présente à sa main pour s'écarter du châtiment qui lui paraît la peine la plus dure qu'il dût souffrir. C'est ce que l'on peut attribuer audit Gilles Levent, qui certainement n’a frappé son supérieur d'un coup de couteau prémédité mais dans un moment d'importunité qui peut arriver à tout le monde.

\section{V - Les collégiens doivent-ils être punis ?}

Si les maîtres sont responsables du désordre, faut-il punir les collégiens ? Dans l'affirmative, quelle peine faut-il leur appliquer? Guels sont les arguments avancés?

Consulté par Saint-Florentin dans l'affaire du jeune de Lattre, l'intendant du Hainaut choisit le parti de l'indulgence. Il propose d'accorder les lettres de rémission demandées par les parents de l'accusé. Elles seraient cependant assorties de 
l'interdiction " de se trouver dans la ville du Quesnoy pendant un certain nombre d'années, pour épargner à la dame Pasqual la vue de l'homicide de son fils ". Pour convaincre Saint-Florentin et obtenir le pardon de l'enfant, il invoque ce qu'il considère comme une faute professionnelle du principal du collège et le jeune âge de l'accusé. Et surtout, il utilise l'argument de la condition sociale et organise la défense de de Lattre à partir du thème de l'honneur offensé.

Dans l'affaire Levent, les documents, plus abondants, montrent que des points de vue différents se sont affrontés. Le subdélégué Hennet et l'intendant de Boisemont retiennent également l'argument de la condition sociale, mais sans en faire comme dans l'affaire de Lattre l'élément décisif pour accorder le pardon. Tous deux font remarquer que Gilles Levent est de "bonne famille roturière ", que son père est "d'une fortune aisée et allié à de très honnêtes gens de famille roturière ". Les remarques sur ce thème s'arrêtent là. À la compréhension de l'un s'oppose le légalisme de l'autre. Pour l'intendant, les circonstances de l'affaire doivent conduire les commissaires royaux à accorder à Gilles Levent les lettres de rémission que son père sollicite. Il faut noter que l'intendant avait, en un premier temps, proposé d'assortir le pardon d'une peine de deux ou trois mois de prison. La partie de la phrase faisant mention de cette peine est raturée dans la minute de la lettre adressée à Saint-Florentin. L'intendant est donc pour le pardon total. Quand il apprend de son subdélégué la peine infligée à Gilles Levent, il ne peut s'empêcher de manifester son indignation en écrivant en haut de la lettre de ce dernier : "Voilà une procédure bien rigoureuse contre un enfant ".

Le subdélégué Hennet, en revanche, estime que Gilles Levent, en refusant de se présenter devant ses juges, ne peut être absous de toute peine. Le délit de contumace le condamne à une peine limitée dans le temps :

Il n'est pas exempt pour cela d'une peine qu'il doit encourir, et qui serait le blâme s'il s'était représenté, et étant ainsi contumace la peine qu'il doit encourir est le bannissement à temps.

Dans la lettre qu'il envoie à l'intendant pour l'informer de la condamnation de Gilles Levent, le subdélégué ne fait aucun commentaire. Pour être complet, on ajoutera qu'en dessous de la remarque de l'intendant jugeant bien dure la peine infligée au jeune homme figure une annotation dont on n'a pu identifier l'origine. Elle témoigne de la plus totale rigueur : " Je ne trouve point ce jugement rigoureux pour un jugement par contumace".

Les commissaires royaux chargés de statuer sur la demande de lettres de rémission ne sont donc pas ralliés à la proposition de l'intendant du Hainaut, ce 
qui aurait eu pour effet de faire cesser les poursuites judiciaires entamées à l'encontre de Gilles Levent. La lettre dans laquelle Saint-Florentin l'informe du rejet de l'octroi de lettres de rémission met en lumière leurs motivations :

Messieurs les commissaires du Conseil nommés par le Roi pour examiner les affaires du sceau ont pensé qu'il était de la plus grande importance de ne pas laisser impunie une révolte d'un écolier contre ses maîtres qui a été poussée au point de blesser le supérieur de la maison avec une arme aussi dangereuse que l'est un couteau et qu'il était nécessaire d'en faire un exemple qui pût contenir les autres écoliers et prévenir de pareils désordres. Si l'accusé eût été prisonnier, peut-être sa grande jeunesse et l'imprudence qu'il y a eu de la part des maîtres auraient-elles pu porter à la traiter avec quelque indulgence. Mais étant contumax, on ne peut rien suppléer en sa faveur.

On voit tout d'abord surgir dans ce passage la crainte des mutineries scolaires, qui, selon Philippe Ariès, cessent à la fin du XVII ${ }^{\mathrm{e}}$ siècle ${ }^{33}$, mais dont le souvenir reste encore vivace. Les " émotions de la jeunesse " sont toujours un objet de crainte suffisant pour inciter le pouvoir à sévir. Pour les commissaires royaux, la nécessité d'une peine exemplaire est une évidence. Enfin, on peut y lire un désaveu de l'attitude conciliante de l'intendant et une approbation de l'attitude plus légaliste de son subdélégué, pour qui le délit de contumace interdit le pardon.

L'étude de ces deux dossiers ne peut conduire à des généralisations hâtives et péremptoires sur la question de la violence dans les rapports entre élèves, ou entre maîtres et élèves, dans les collèges d’Ancien Régime. Les affaires de Lattre et Levent illustrent la vertu de l'événement. Au-delà du simple fait divers, leurs suites judiciaires sont à l'origine de la production d'archives mettant à nu des pratiques, des comportements et des choix éducatifs qui, sans elles, resteraient inaccessibles à l'historien. Comme le note fort justement Marie-Madeleine Compère, le dossier de Lattre nous rappelle la nécessité " de replacer dans leur contexte social et mental les mœurs scolaires " ${ }^{34}$. De son côté, le dossier Levent, mais aussi les lettres des parents d'élèves du collège de Lille, montrent que les châtiments corporels ne sont pas démodés dans la seconde moitié du XVIII ${ }^{\mathrm{e}}$ siècle et qu'ils ne subsistent pas seulement « dans les provinces reculées, dans les écoles obscures où la pédagogie

33 Philippe Ariès, L'Enfant et la vie familiale..., op. cit., p. 356.

34 Marie-Madeleine Compère, Du collège au lycée..., op. cit., p. 218. 
des lumières n'a pas encore pénétré» ${ }^{35}$. Leur abolition officielle en 1803 ne les fera pas disparaître ${ }^{36}$. Les témoignages littéraires, les archives de la série $\mathrm{F}^{17}$ des Archives nationales et de la série T des Archives départementales, montrent que beaucoup de professeurs et de surveillants ont encore la main leste. Au XIX ${ }^{\mathrm{e}}$ siècle, la violence fait toujours partie des méthodes pédagogiques ${ }^{37}$.

Ces deux affaires sont intéressantes dans ce qu'elles disent des réactions des personnalités extérieures à l'institution scolaire. Globalement, celles-ci sont d'accord pour condamner le geste des deux adolescents. En même temps, elles cherchent à comprendre leurs motivations. Cela les conduit à blâmer les responsables des collèges, d'une part un principal qui tolère que les pensionnaires aient des couteaux, de l'autre un supérieur qui entend sanctionner une faute légère par la peine des verges. Enfin, ces deux affaires montrent la diversité de leur regard sur la nature de la peine que la justice doit prononcer. Si les autorités locales sont portées à l'indulgence, le pouvoir central rappelle qu'on ne peut tolérer la révolte d'un écolier contre ses maîtres et que tout acte remettant en cause l'ordre établi doit être puni.

Philippe MARCHAND

Université Charles-de-Gaulle-Lille III pmarchand356@numericable.fr

35 Jean de Viguerie, L'Institution des enfants..., op. cit., p. 249-250 : "Après 1750, les châtiments corporels sont tout à fait démodés. On ne fouette plus que dans les provinces reculées, dans les écoles obscures où la pédagogie des lumières n’a pas encore pénétré [...] Les collèges des grandes villes ne les connaissent plus ". Une lettre écrite par le précepteur de deux jeunes Lillois pensionnaires au collège de Navarre (Paris) montre au contraire que, dans cet établissement, le sous-principal fait encore usage de la férule : "Croiriez-vous ", écrit le précepteur au père des enfants, "que Villerval, l'enfant du monde le moins fait certainement pour mettre le désordre quelque part, et bien croiriez-vous que cet imbécile d'homme, dans un petit tumulte qui est arrivé au réfectoire, l'a été choisir de préférence pour en faire un exemple qui effrayât la multitude ? " : AD Nord, Ê 2294/116, Vallier à Fruict, Paris, 15 janvier 1781. L'enfant parvient à s'échapper et court se réfugier dans la chambre de son précepteur.

36 Arrêté du 21 prairial an XI [10 juin 1803] organisant la vie quotidienne des écoles secondaires et des lycées, art. 122.

37 Jean-Claude Caron, À l'école de la violence. Châtiments et sévices dans l'institution scolaire au XIX ${ }^{e}$ siècle, Paris, Aubier, 1999 (Collection historique). 


\section{Annexe}

\section{Lettres d'ester à droit ${ }^{1}$ pour Gilles Levent, 24 octobre 1770}

Louis, par la grâce de Dieu, roy de France et de Navarre : au Prévot royal juge civil et criminel des villes et prévôté de Bavay et à tous nos autres officiers et justiciers qu'il appartiendra, salut. Notre amé Gilles Levent nous a fait remontrer qu'en mil sept cent cinquante huit, tems auquel il était écolier dans le collège des prêtres de l'oratoire de la ville de Bavay en Flandres, un des écoliers faisant fonction de censeur dans la seconde classe, appelée le Musoeum, où se tiennent les écoliers nommés poëtes, dénonça au régent le nommé Pierre Prévot, autre écolier de la même classe, pour pétulance. Ce régent le condamna à recevoir le fouet, ce qui était contre l'usage et règles de ce collège, où l'on ne se sert plus de ce genre de punition envers des écoliers appelés poëtes ; que tous les écoliers se récrièrent contre cette punition et ce renversement des usages et des privilèges qu'avaient les élèves de cette classe de se regarder comme exempts de cette punition ; quoique l'exposant ne se fut pas distingué d'entre les autres dans cette protestation générale, le père Cordier le choisit pour le condamner au susdit châtiment et voulut le lui donner lui-même en l'invectivant des injures les plus grossières, ce qui irrita si fort cet enfant qu'il refusa et demanda qu'on le laissât plutôt sortir du collège. Le père Cordier, au lieu de déférer à cette demande, envoya appeler le père de Glines supérieur dudt. collège, qui étant arrivé avec plusieurs autres pères fut assez imprudent pour irriter encore cet enfant et lui donner plusieurs coups ; celui-ci voyant tous ces pères se jeter sur lui se retira précipitamment, montant sur la table, sur laquelle les écoliers écrivent ordinairement et s'éloignant toujours des assaillants ; mais le père de Glines poussa la violence et l'imprudence jusqu'à saisir l'exposant par la jambe et le frappant durement contre la table, non seulement il lui causa la plus grande douleur et la lui blessa en plusieurs endroits, mais encore voulut le faire tomber de dessus la table par terre. L'exposant qui avait inutilement demandé à sortir du collège ainsi qu'une grande partie des écoliers, se sentant entraîné et au moment d'être précipité, voulut repousser le père de Glines et eut le malheur de le frapper à l'épaule droite de son couteau que par hasard il avait dans ce moment à la main : cet accident ayant fait cesser le combat, l'exposant se sauva par-dessus les murs du collège et fut se cacher dans une maison voisine où il se fit panser les blessures qu'il avait à la jambe : de là il se retira dans la maison paternelle. Quoique la blessure du père de Glines fût si légère qu'elle n'eut aucune suite et qu'elle n'empêcha pas même ce supérieur de faire ses fonctions ordinaires, notre procureur de la prévôté de Bavay crût devoir rendre plainte le lendemain et demanda permission d'informer, ce qui luy fut accordé le vingt-six octobre mil sept cent cinquante huit. Les informations furent faites ; le père de Glines ne fut pas même entendu ; le père Cordier dont l'imprudence était

1. Un condamné par contumace devait, au-delà d'un délai de cinq ans, demander une permission pour introduire une action en justice (ester à droit) de façon à présenter sa défense et purger sa peine : c'est cette permission qu'accordaient les lettres d'ester à droit. 
évidente chercha dans sa déposition à la pallier ; au lieu de faire entendre plusieurs écoliers, on n'en appela qu'un seul qui déclara publiquement que cet accident n'était arrivé que par l'effet de la violence exercée contre l'exposant et la colère dans laquelle on l'avait mis, et que cette punition était contre les usages. Cependant les parents de l'exposant intimidés par l'appareil de cette procédure criminelle crurent devoir le faire disparaître et passer en païs étranger, de manière que par contumace l'exposant a été condamné aux galères à perpétuité par sentence du trois mars mil sept cent cinquante neuf. Ce qui a été exécuté par effigie. L'exposant qui appartient à une famille honnête et estimée dans le pays et qui d'ailleurs depuis ce tems a tenu la conduite la plus régulière, se voit avec peine hors d'état de purger la contumace, les cinq années étant plus que passées depuis le trois mars mil sept cent cinquante neuf ; mais comme la punition est visiblement disproportionnée au délit qui n’a été occasionnée que par l'imprudence des régens et qui méritait au plus une légère réprimande proportionnée à l'âge et à l'état qu'avait alors l'exposant, il désire pouvoir purger la contumace, et faire parvenir sa justification par les voies ordinaires, ce qu'il ne peut faire sans nos lettres, sur ce (...) qu'il nous a très humblement supplié de luÿ accorder. A ces causes voulant traiter favorablement l'exposant, nous vous mandons par les présentes signées de notre main, que notre Procureur et autres qu'il appartiendra appeler par devant vous, s'il vous appert de ces défauts et contumace par l'ignorance et la crainte où était l'exposant des poursuites qui se sont faites contre luy, vous ayez à le recevoir à ester à droit et à se justifier des faits à luy imputés, tout ainsÿ qu'il eût pu faire avant votre jugement du trois mars mil sept cent cinquante neuf, que nous voulons ne lui nuire ni préjudicier pour ne s'être présenté dans les cinq ans portés par nos ordonnances, dont nous l'avons de notre grâce spéciale, pleine puissance et autorité royale relevé et relevons par ces présentes, à la charge de se mettre en état lors de la présentation d'icelles de résoudre les dépens des contumaces. Mandons au premier notre huissier ou sergent sur ce requis de faire pour l'exécution des présentes tous exploits et autres actes de justice requis et nécessaires ; de ce faire luÿ donnons pouvoir. Car tel est notre bon plaisir. Donné à Fontainebleau le vingt quatrième jour d'octobre de l'an de grâce mil sept cent soixante dix, et de notre règne, le cinquante sixième.

Louis

par le Roi

Le Duc de Choiseul

Lettres d'ester à droit pour Gilles Levent, 24 octobre 1770 (publiées par Henri Bièvelet, "Notes sur le couvent des Récollets et l'Oratoire de Bavai au XVIII siècle ", Annales du Cercle archéologique de Mons, 1950-1953, p. 95-110). 